\title{
Sustentabilidad como respuesta a una urgencia
}

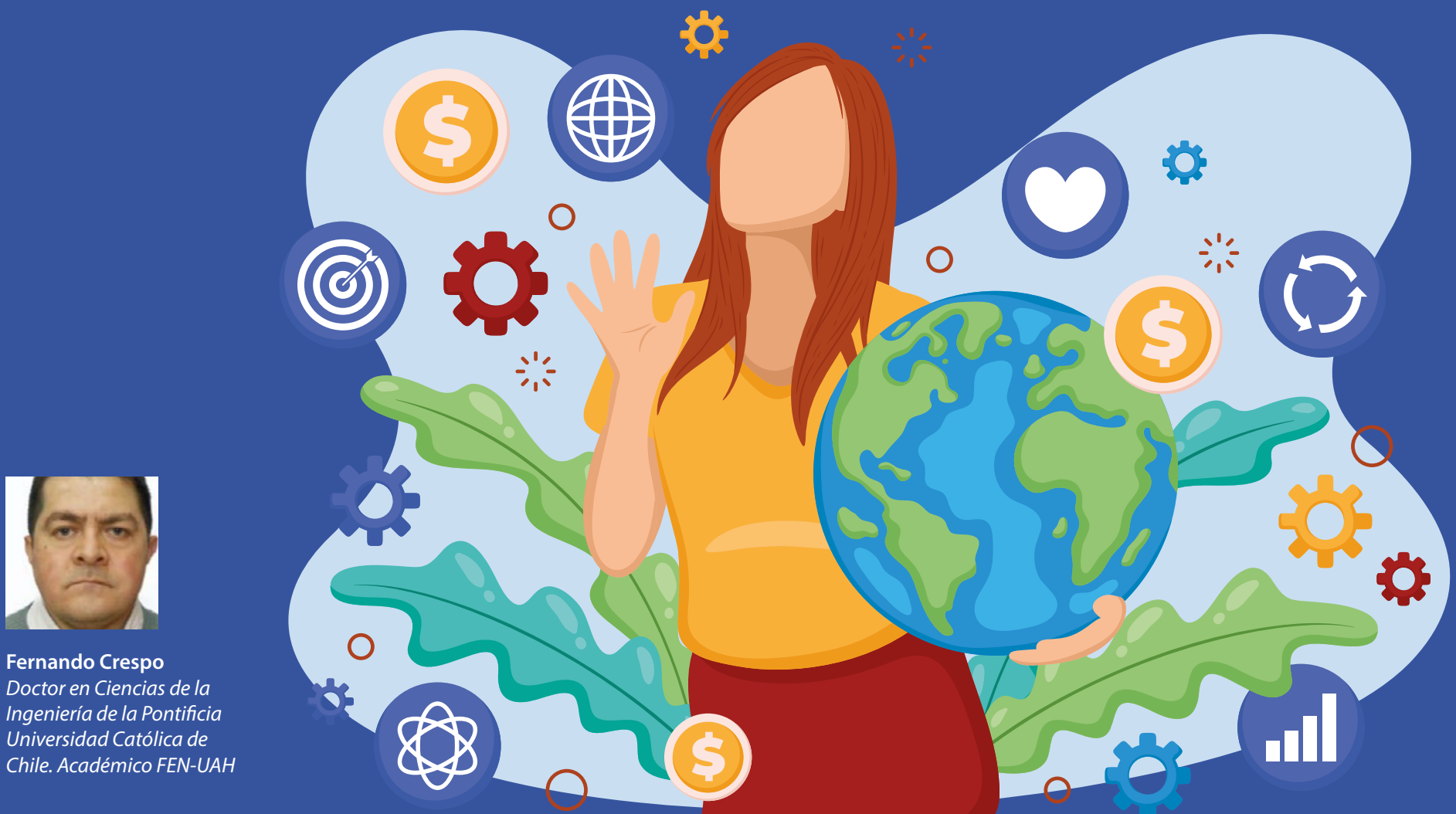

Existe una serie de informes que alertan sobre las consecuencias que tendrán tanto el cambio climático como el resultado del patrón vigente en la operación económica global, los que revelarían un conjunto de externalidades negativas a partir del año 2030. Considerando ese horizonte, en este documento, se propone la sustentabilidad como una estrategia más amplia, que permita anteponerse a escenarios que escapan a la normativa medioambiental y nos permitan generar una respuesta resiliente a los impactos futuros, considerando que ya es posible advertir esta dimensión en casos incipientes a nivel global.

\section{Un futuro incierto}

A Ivin Tofler escribió el shock del futuro en 1969 (Toffler, 1973), y una de sus mayores inquietudes era buscar las voces fuera de corriente para visualizar las macrotendencias del futuro, y como los seres humanos estábamos sufriendo una serie de síntomas psicológicos asociados a demasiados cambios en un tiempo muy breve. Leerlo hoy nos parecería un libro premonitorio respecto de las tecnologías por venir y sus impactos. La pregunta es cuál es la estrategia que nos permitiría mejor enfrentar los tiempos que vienen. Yuval Harari, tampoco se ha quedado atrás respecto del impacto del desarrollo tecnológico en el futuro (Harari, 2016). Peter Turchin predijo que el 2020 iba a ser turbulento socialmente diez años antes, a partir del análisis de los patrones de ciclos históricos mediante modelos matemáticos (Turchin, 2020). Con una inestabilidad creciente a contar de la fecha, lo anterior con el mérito de ser el único investigador que preveía ese escenario, en un grupo consultado por la revista Nature.

Hemos vivido una pandemia mundial a raíz de la proliferación de un coronavirus. El ba- lance a un año del inicio de la Pandemia por COVID-19, con consecuencias cuyo impacto aún no han sido medidas, muestra contracciones en todas las economías, con la excepción de China, lo que acarrea problemas sociales de compleja solución y urgente atención, como la creación de empleo, entre otros efectos (Menz, 2021), y el consiguiente endeudamiento de los países para sortear la crisis, unido a caídas en importaciones y exportaciones, lo que dificultará la recuperación de los países. Todo lo anterior en lo inmediato. Sin mediar, que la crisis sanitaria no ha impedido protestas en todo el globo. 
El último informe del Grupo Intergubernamental de Expertos sobre el Cambio Climático, no agrega un mejor escenario, pues alerta de un calentamiento real al año 2021 de $1.0^{\circ} \mathrm{C}$, con perspectiva de alcanzar los $1.5^{\circ} \mathrm{C}$ entre 2030 y 2052 si continúa aumentando al ritmo actual, con efectos de fenómenos climáticos y meteorológicos extremos, contabilizados desde 1950, además de cambios en ecosistemas terrestres y oceánicos, y de los servicios que prestan, con riesgos inminentes para la humanidad (IPCC, 2021).

Otra investigación, en la actualidad ignorada, plasmada en 1972 en el libro Los límites al crecimiento (Meadows et al., 1972), utilizando una técnica de simulación basada en dinámica de sistemas, el equipo de investigadores elaboró un informe que llega a la conclusión que el incremento de la población mundial, la industrialización, la contaminación, la producción de alimentos y la explotación de recursos naturales si se mantiene sin variación llevaría a un colapso en 100 años a contar de la fecha del informe. El informe ha sido actualizado con posterioridad (2004), donde se expone que no puede seguir existiendo un crecimiento poblacional, económico e industrial ilimitado en un planeta de recursos limitados. El mismo informe establece que es posible modificar las tasas de desarrollo para alcanzar estabilidad ecológica, sostenible, a largo plazo. La condición es que el estado de equilibrio debe ser global.

La dinámica de sistemas es una estrategia de simulación que fue desarrollada por el profesor Jay Forrester en el Massachusetts escenario más probable muestra un patrón de colapso, dentro de una década (2030), y el caso optimista muestra un declive suave para los próximos años, desde la fecha actual.

La conclusión es que nos esperan años difíciles. La evidencia es sustantiva, respecto de la afectación en el corto o mediano plazo a cambios que influenciarán en nuestras vidas, ya sea por la presión que aumentará para reducir los gases de efecto invernadero, lo que tendrá implicancias e impactos tecnológicos, como los efectos locales del cambio climático, que ya son percibidos por la población, unidos a escenarios de crecimiento económico volátil. En cualquier caso, no habría forma de evitar esas experiencias posibles.

\section{Sustentabilidad: una alternativa al colapso}

Ante los escenarios descritos, existe aún una estrategia posible para minimizar los impactos, la sustentabilidad surge como una respuesta imperativa, positiva y alentadora. La World Commission on Environment and Development la definió en el informe Brundtland, "Our Common Future", de 1987 como: "El desarrollo sustentable hace referencia a la capacidad que haya desarrollado el sistema humano para satisfacer las necesidades de las generaciones actuales sin comprometer los recursos y oportunidades para el crecimiento y desarrollo de las generaciones futuras".

La definición implica satisfacer necesidades de la generación actual, sin perjudicar

\section{"El límite de crecimiento y el cambio climático, plantean a nivel personal, desafíos que incluyen modificaciones de comportamiento y actitud incluso en la dieta, ya sea por la dificultad de conseguir
algunos alimentos, como por el daño que generan otros"}

Institute of Technology, quién planteó originalmente el estudio del colapso a través de un modelo que denominó Dinámica Mundial, que fue posteriormente perfeccionado por Meadows y su equipo. Herrington (2020), responsable de análisis de sistemas dinámicos y sustentabilidad de KPMG, actualizó el estudio Forrester y Meadows a datos del año 2020. Para lo cual se crearon diferentes escenarios, donde el sidades tienen sentido de ser satisfechas, y en caso de tenerlo, si lo estamos haciendo con el menor impacto medioambiental y energético posible. Respuestas a estas preguntas, requieren cambios culturales y educativos en los patrones de consumo, en países desarrollados ya han abierto el mercado a empresas que trazan su huella de carbono, su huella de agua, o sus procesos o sus materias primas son eco sustentables o amigables al medio ambiente, o producción responsable. Un ejemplo, es la difusión de Mercado Social en Madrid, plataforma de visualización para consumidores de empresas que usan la economía social y solidaria, de manera de contribuir a la sostenibilidad (https://madrid.mercadosocial.net/). Un ejemplo de contribución al reciclaje con apoyo de consumidores en Chile, es la empresa que desarrolla vasos desde botellas recicladas Mawka (http:// www.mawka.cl) incorporando diseño.

\section{Nuestro aporte individual}

El límite de crecimiento y el cambio climático, plantean a nivel personal, desafíos que incluyen cambios de comportamiento y actitud incluso en la dieta, ya sea por la dificultad de conseguir algunos alimentos, como por el daño que generan otros. Un ejemplo de lo anterior es el efecto que genera el sector ganadero. Por ejemplo, es el responsable del $44 \%$ de la emisión de gas metano por actividades realizadas por el hombre (Gerber, et al, 2013), un cambio en la demanda de carne, eventualmente cambiaría el nivel de producción ganadera, sin dejar de considerar las políticas de mitigación que el sector implemente a su actividad. No nos hemos preguntado, ¿pero cuánto impacto ambiental genera un asado?

Por otra parte, la sustentabilidad nos cuestiona respecto de nuestra economía doméstica, el cambio climático está relacionado fuertemente con la generación de energía, y la energía es un componente esencial para el desarrollo económico (Asghar, 2008). Es un costo esencial para la producción y un recurso limitado. De esa forma, es ineludible plantear el uso eficiente de energía en los domicilios, mejorar la eficiencia térmica de las habitaciones, reducir al máximo el consumo de combustibles fósiles para proveer la energía para hacer habitable una casa. Interesante para este aspecto es el plan del Programa de Protección Patrimonio Familiar (PPPF) 
del Ministerio de Vivienda y Urbanismo de Chile (MINVU, 2018), que financia proyectos de mejora de aislación, aislación térmica o instalación de calefactores solares en viviendas, aspectos todos que llevan a una mejora de calidad de vida, y disminución de costos mensuales por uso alternativo de energías.

\section{Organizaciones}

Y por último a nivel de sector industrial, cualquiera sea su área, servicios o manufactura, la sustentabilidad hoy debería considerarse al menos con dos ejes, por una parte responder a la legislación vigente, posibles cambios futuros de esta y una población con mayor consciencia medioambiental, donde cada uno de estos aspectos será cada vez más exigente respecto de sus cumplimientos, esto le llamaremos el eje medioambiental por una parte, y por otra, de un eje, estratégico y prospectivo que deberá velar porque la empresa siga subsistiendo aún dentro de márgenes acotados de actividad, como lo que ha pasado durante la pandemia del Covid-19, lo que obliga a una racionalización y conceptualización futura de los recursos disponibles para y por la empresa. Muy probablemente, en la actualidad, las direcciones estratégicas no incorporan este punto, pero al menos, es urgente plantearlo frente a un ecosistema empresarial que de seguir bajo los mismos parámetros actuales en algún momento presente caídas globales en su actividad, lo que puede poner en riesgo el funcionamiento del negocio mismo, su inversión y su posterior supervivencia, le llamaríamos la sustentabilidad como preparación al shock del futuro.
Uniendo ambos ejes, Amazon, ya tiene la autorización para utilizar drones en la entrega de productos. Ha confirmado pedidos para la incorporación de flotas de camionetas eléctricas en Europa, con el fin de ser neutral a la huella de carbono para el año 2040, y transformar su flota de transporte en una de las más sostenibles del mundo. Pensar que dicha política solo apunta al eje medioambiental, olvida la capacidad de seguir operando en caso de alteraciones de la cadena de suministro y la ausencia de combustibles fósiles, el poseer una flota eléctrica le garantiza operatividad, hace su cadena de abastecimiento más resiliente, aún bajo ciertos riesgos extremos, garantizando la entrega en la última milla, o envíos de corta distancia. Este tipo de visualización estratégica es necesaria para el corto plazo, toda vez que no habrá más de 10 años para tener una respuesta en desarrollo.

Las universidades tampoco son ajenas al desafío de la sustentabilidad, hay una búsqueda permanente de equilibrio entre sus fines que la inspira, y los recursos disponibles (Montes, 2018).

Temas interesantes respecto de respuesta sustentable a la operación de una empresa, tendrá que ver con el impacto medioambiental asociada al teletrabajo, desconozco investigaciones en esa línea, pero de producirse ahorros significativos en las empresas y de lograr disminuciones en la huella de carbono, y otras externalidades positivas, el teletrabajo puede transformase en un herramienta más para la sustentabilidad medioambiental y empresarial del planeta, y convertirse en una aliada para generar empleos en escenarios adversos.

\section{El Estado}

Un elemento adicional, es que la operación del Estado debería procurar también ser sustentable. Su operación no es neutral de carbono, y por tanto, también merece ser revisada en aras de lograr un mejor punto de equilibrio con la menor cantidad de impacto al medio ambiente. En el pasado, hay ejemplos en todo el mundo de operaciones llevadas a cabo por Estados o Gobiernos con consecuencias desastrosas para el medioambiente.

\section{Reflexión final}

La sustentabilidad hoy no es la meta, es un marco conceptual que nos provee una orgánica, una forma de concebir el mundo, para responder a los próximos años, con el fin de revertir y mitigar los efectos que la actividad humana ha causado al planeta, y plantear la racionalización de recursos presentes y futuros, de forma amigable a la Tierra, pero también resilientes a variaciones económicas severas. Podría discutirse, que lo anterior es un punto de vista pesimista, pensando en la tecnología por desarrollar y por llegar va a jugar un papel importante en términos de mejora de eficiencia y mitigación, pero tal como menciona Herrington (2020), entre el desarrollo y la implementación masiva de una tecnología hay un tiempo de rezago, lo que atrasa el ciclo positivo de su aplicación. El futuro está en nuestras decisiones, incluyendo las microdecisiones (Figueres and RivettCarnac, 2021). La sustentabilidad es una forma de responder a emergencias futuras.

Bibliografía

- Asghar, Zahid (2008). Energy-gdp relationship: a causal analysis for the five countries of South Asia. Applied Econometrics and International Development. Vol. 8-1.

- Gerber, P.J., Steinfeld, H., Henderson, B., Mottet, A., Opio, C., Dijkman, J., Falcucci, A. \& Tempio, G. 2013. Enfrentando el cambio climático a través de la ganadería Una evaluación global de las emisiones y oportunidades de mitigación. Organización de las naciones unidas para la alimentación y la agricultura (FAO), Roma.

- $\quad$ Figueres, Christiana; Rivett-Carnac, Tom (2021). El futuro por decidir. Editorial Debate.

- Harari, Yuval (2016). Homo Deus: Breve historia del mañana. Editorial Debate.

- Herrington, Gaya (2020). Update to limits to growth: Comparing the World3 model with empirical data. Journal of Industrial Ecology. https://doi.org/10.1111/ jiec.13084. Una publicación preliminary está disponible en https://advisory.kpmg.us/articles/2021/limits-to-growth.html [20-08-2021].

- IPCC (2021). Resumen para responsables de política. Calentamiento Global de $1.5^{\circ} \mathrm{C}$. En https://www.ipcc.ch/site/assets/uploads/sites/2/2019/09/IPCC-SpecialReport-1.5-SPM es.pdf [20-08-2021].

- Meadows, D. H., Meadows, D. L., Randers, J., \& Behrens, W. W (1972). Los límites del crecimiento: informe al Club de Roma sobre el predicamento de la humanidad. México: Fondo de Cultura Económica.

- Menz, Eduardo (2021). Impactos Económicos y Sociales a un año de la Pandemia por COVID-19 en América Latina y el Caribe. Flacso-Chile, Marzo 2021. En https:// flacsochile.org/doc/2021/Doc_Electronico_Impactos_Economicos_Sociales_a_un_a\%C3\%B10_de_la_pandemia_covid_19_ALC.pdf [20-08-2021]

- MINVU (2018). Programa de Protección del Patrimonio Familiar. https://bonosdelgobierno.com/primer-llamado-al-subsidio-del-programa-de-proteccion-delpatrimonio-familiar-2018/ [20-08-2021].

- $\quad$ Montes SJ, Fernando (2018). Pensando la Universidad. Santiago: Editorial Universidad Alberto Hurtado.

- Toffler, Alvin (1973). El shock del Futuro. Barcelona: Plaza \& Janes, S.A.

- Turchin, Peter (2020). The Science behind My Forecast for 2020. http://peterturchin.com/cliodynamica/the-science-behind-my-forecast-for-2020/ [20-08-2021]. 